\title{
Texture and Lamellae Distribution Functions in Lamellar Eutectics
}

\author{
H. J. BUNGE \\ Institut für Metallkunde und Metallphysik, Technische Universität Clausthal \\ (FRG)
}

(Received May, 3 1986)

The crystallographic orientation distribution and the geometrical lamellae orientation distribution in lamellar eutectics are, in general, not independent of each other. The combined orientation-lamellae distribution function depends on five angular parameters. X-ray diffraction in such eutectics may exhibit an anisotropic macroscopic absorption factor if the penetration depth of the X-rays is large compared with their planar size. As a consequence, the reflected X-ray intensity may depend on a third angle $\gamma$, i.e. a rotation of the sample about the diffraction vector $s$ additionally to the usual pole figure angles $\alpha, \beta$ which describe the orientation of the diffraction vector $s$ with respect to the sample coordinate system. It is thus necessary to measure three-dimensional generalized pole figures instead of conventional two-dimensional ones.

\section{GENERAL DEFINITIONS}

In order to describe the orientation distribution in binary lamellar eutectics the lamellae orientation distribution has to be considered along with the crystal orientation distributions in both phases. In order to describe these orientation distributions we fix a coordinate system $K_{A}$ in the sample and a system $K_{B}^{\alpha}$ in each crystal of the phase $\alpha$. The orientation of the crystal is then described by the rotation $g^{\alpha}$ which transforms $K_{A}$ into $K_{B}^{\alpha}$, Figure 1.

$$
K_{B}^{\alpha}=g^{\alpha} \cdot K_{A}
$$



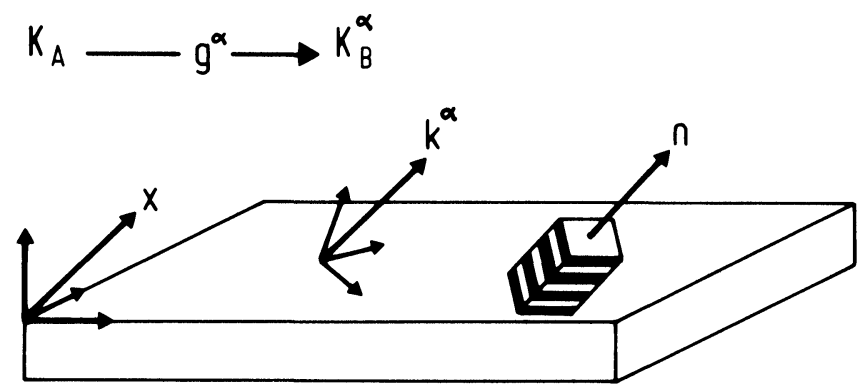

FIGURE 1 The sample coordinate system $K_{A}$, the crystal coordinate system $K_{B}^{\alpha}$, the crystal orientation $g^{\alpha}$ and the lamellae orientation $n$.

A crystal direction $k^{\alpha}$ is then parallel to the sample direction $x$ so that

$$
x=g^{\alpha} \cdot k^{\alpha}, \quad x_{i}=g_{i j}^{\alpha} \cdot k_{j}^{\alpha}
$$

The orientation distribution in the lamellar eutectic is defined by the volume fractions of crystals having the orientation $g^{\alpha}$ and the normal direction $n$ to the lamellae plane. The normal direction can be referred to the sample coordinate system or to the crystal coordinate system, Figure 1. Hence, the orientation distribution in the phase $\alpha$ can be described by the two equivalent but complementary functions

$$
\begin{aligned}
& \frac{d V^{\alpha}}{V^{\alpha}}=F^{\alpha}(g, x) d g d x \\
& \frac{d V^{\alpha}}{V^{\alpha}}=\phi^{\alpha}(g, k) d g d k
\end{aligned}
$$

where $V^{\alpha}$ is the volume fraction of the phase $\alpha$ such that

$$
V^{1}+V^{2}=1
$$

The two functions $F^{\alpha}$ and $\phi^{\alpha}$ are related to each other according to Eq. (2)

$$
\begin{array}{r}
F^{\alpha}(g, g \cdot k)=\phi^{\alpha}(g, k) \\
\phi^{\alpha}\left(g, g^{-1} \cdot x\right)=F^{\alpha}(g, x)
\end{array}
$$

The textures in the usual sense are obtained by integration over $x$ 
or $k$

$$
\begin{aligned}
& f^{\alpha}(g)=\int F^{\alpha}(g, x) d x \\
& f^{\alpha}(g)=\int \phi^{\alpha}(g, k) d k
\end{aligned}
$$

The lamellae distribution functions are obtained by integration over $g$

$$
\begin{aligned}
L^{\alpha}(x) & =\int F^{\alpha}(g, x) d g \\
\Lambda^{\alpha}(k) & =\int \phi^{\alpha}(g, k) d g
\end{aligned}
$$

The lamellae orientation distribution functions $L^{\alpha}(x)$ in both phases are assumed to be identical

$$
L^{1}(x)=L^{2}(x)
$$

This relationship is not strictly necessary but will hold, for example, if one assumes a constant and orientation independent lamellae thickness in both phases. A similar relationship is, of course, not required for the crystallographic lamellae distribution function $\Lambda(k)$.

In the following we shall restrict our considerations mostly to one of the phases. The upper index $\alpha$ will then be omitted. Particular axis distribution functions of the functions $F$ and $\phi$ can be defined which describe the volume fraction of crystals, the crystal direction $h$ of which is parallel to the sample direction $y$ while the lamellae normal direction is either $x$ or $k$.

$$
\begin{aligned}
& D(h, y, x)=\frac{1}{2 \pi} \int_{h \| y} F(g, x) d \psi \\
& \Delta(h, y, k)=\frac{1}{2 \pi} \int_{h \| y} \phi(g, k) d \psi
\end{aligned}
$$

They can be interpreted as pole figures or inverse pole figures depending on whether $h$ or $y$ is kept constant. The total axis distribution function is then obtained by

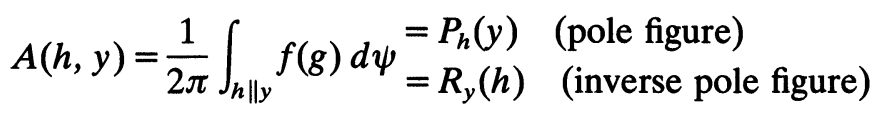


It is the integral over the particular axis distribution functions

$$
A(h, y)=\int D(h, y, x) d x=\int \Delta(h, y, k) d k
$$

The functions are assumed to be normalized in multiples of the random density (mrd-units). This means

$$
F_{r}=\phi_{r}=f_{r}=L_{r}=\Lambda_{r}=D_{r}=\Delta_{r}=A_{r}=1
$$

where $r$ indicates the random distribution. The orientation element $d g$ and the solid angular elements $d x$ and $d k$ are defined such that

$$
\int d g=\int d x=\int d k=1
$$

The first part of this definition agrees with the definition used formerly (Bunge, 1982) whereas the second and third part deviate from the formerly used definitions by the factor $1 / 4 \pi$. It seems, however, more convenient to use the analogous definition in the space of orientations $g$ and in the space of directions $x$ or $k$. Since the functions defined above are distribution functions their integrals over the whole orientation space are always identical with the corresponding integrals over the random functions. Hence, it is

$$
\begin{aligned}
& \int f(g) d g=1 \\
& \int L(x) d x=\int \Lambda(k) d k=\int P_{h}(y) d y=\int R_{y}(h) d h=1 \\
& \int F(g, x) d g d x=\int \phi(g, k) d g d k=1
\end{aligned}
$$

The sample is assumed to have the symmetry $G^{s}$ with the symmetry operations $g^{s}$ and $u^{s}$ (symmetry rotations and inversions). This symmetry is symbolized by one dot on top of the functional symbol

$$
G^{s}=\left\{g^{s}, u^{s}\right\}
$$

The crystals have the symmetry $G^{c}$ with the operations $g^{c}$ and $u^{c}$. This symmetry is symbolized by two dots

$$
G^{c}=\left\{g^{c}, u^{c}\right\}
$$




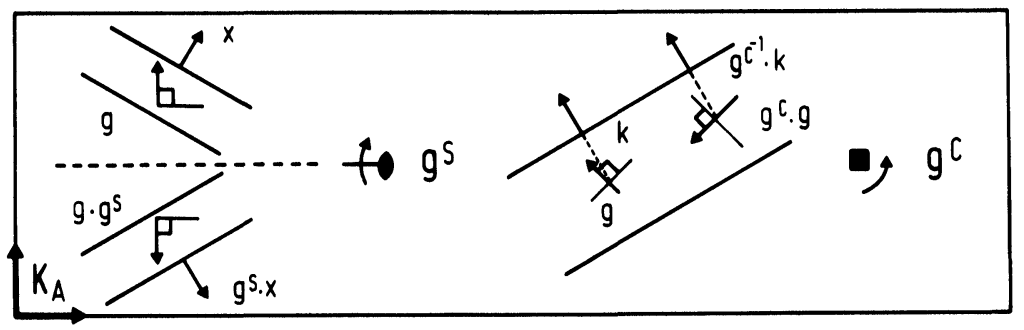

FIGURE 2 Influence of crystal symmetry $g^{c}$ and sample symmetry $g^{s}$ on the crystal orientation $g$ and the lamellae normal direction $x$ in the sample coordinate system and $k$ in the crystal coordinate system.

Finally, the shape symmetry of the lamellae is to be considered. It is an axial symmetry $D_{\infty h}$. When the lamellae are considered in the crystal coordinate system, a reduced crystal symmetry can be defined which contains the operations common to $G^{c}$ and $D_{\infty h}$. This symmetry may be characterized by

$$
G^{\prime c}=\left\{g^{\prime c}, u^{\prime c}\right\}, \quad \text { (吕) }
$$

In the most general cases when the lamellae are not in special orientation with respect to the crystal lattice then this symmetry is triclinic ( 1 or $\overline{1}$ respectively). In other cases it may be higher.

The defined distribution functions must obey the crystal and sample symmetry in the following ways, Figure 2.

$$
\begin{gathered}
\dot{F}\left(g^{c} \cdot g \cdot g^{s}, g^{s} \cdot x\right)=\ddot{F}(g, x) \\
\ddot{\phi}\left(g^{c} \cdot g \cdot g^{s}, g^{c^{-1}} \cdot k\right)=\ddot{\phi}(g, k) \\
\dot{f}\left(g^{c} \cdot g \cdot g^{s}\right)=\dot{f}(g) \\
\dot{L}\left(g^{s} \cdot x\right)=\dot{L}(x) \\
\dot{\Lambda}\left(g^{c} \cdot k\right)=\dot{\Lambda}(k) \\
\dot{D}\left(g^{c} \cdot h, g^{s} \cdot y, g^{s} \cdot x\right)=\dot{D}(h, y, x) \\
\dot{\Delta}\left(g^{c} \cdot h, g^{s} \cdot y, g^{c} \cdot k\right)=\dot{\Delta}(h, y, k) \\
\dot{A}\left(g^{c} \cdot h, g^{s} \cdot y\right)=\dot{A}(h, y)
\end{gathered}
$$

Furthermore, positive and negative lamellae normal direction 
cannot be distinguished. Hence, it is also necessary

$$
\begin{aligned}
& F(g,-x)=F(g, x) \\
& \phi(g,-k)=\phi(g, k)
\end{aligned}
$$

Besides the general case, defined by Eq. (3) we consider two specific cases of texture and lamellae orientation distribution:

In the first specific case it is assumed that texture and lamellae orientation are independent of each other. Then the functions $F$ and $\phi$ take on the specific form

$$
\begin{aligned}
& F(g, x)=f(g) \cdot L(x) \\
& \phi(g, k)=f(g) \cdot \Lambda(k)
\end{aligned}
$$

Because of the relationship Eq. (5) between the two functions $F$ and $\phi$ we obtain with eq. (7)

$$
\begin{aligned}
& L(x)=\int \phi\left(g, g^{-1} \cdot x\right) d g \\
& \Lambda(k)=\int F(g, g \cdot k) d g
\end{aligned}
$$

from which it follows with Eq. (24)

$$
\begin{aligned}
& L(x)=\int f(g) \cdot \Lambda\left(g^{-1} \cdot x\right) d g \\
& \Lambda(k)=\int f(g) \cdot L(g \cdot k) d g
\end{aligned}
$$

The particular axis distribution functions take on the form

$$
\begin{aligned}
& D(h, y, x)=A(h, y) \cdot L(x) \\
& \Delta(h, y, k)=A(h, y) \cdot \Lambda(k)
\end{aligned}
$$

This case of independent lamellae and orientation distribution may be further specialized assuming that all the lamellae are perpendicular to the sample direction $x_{0}$. The lamellar distribution function $L(x)$ then takes on the form

and Eq. (24a) reads

$$
L(x)=\delta_{x x_{0}}
$$

$$
F(g, x)=f(g) \cdot \delta_{x x_{0}}
$$


Replacing $L(g \cdot k)$ in Eq. (26) by Eq. (28) one obtains for the crystallographic lamellae distribution function in this case:

$$
\Lambda(k)=\int_{k \| x_{0}} f(g) d \psi=A\left(k, x_{0}\right)=R_{x_{0}}(k)
$$

The lamellae distribution function $\Lambda(k)$ is then the inverse pole figure of the sample direction $x_{0}$.

In the second specific case it is assumed that the lamellae are perpendicular to one specific crystal direction $k_{0}$ and its crystallographically symmetrical directions. Hence it is

$$
\Lambda(k)=\sum_{g^{c}} \delta_{k, g^{c} \cdot k_{0}}
$$

The crystals with lamellae perpendicular to $k_{0}$ (and not to any other symmetrically equivalent direction $g^{c} \cdot k_{0}$ ) may have the orientation distribution function $\phi^{k_{0}}(g)$. This function must have sample symmetry but it may possess only the reduced crystal symmetry defined in Eq. (17).

$$
{\stackrel{\circ}{\phi^{k}}}^{k_{0}}\left(g^{\prime c} \cdot g \cdot g^{s}\right)=\phi^{k_{0}}(g)
$$

The function $\phi(g, k)$ then takes on the form:

$$
\phi(g, k)=\frac{1}{N^{c}} \sum_{g^{c}} \phi^{g^{c} \cdot k_{0}}(g) \cdot \delta_{k, g^{c} \cdot k_{0}}
$$

which can also be written:

$$
\phi(g, k)=\frac{1}{N^{c}} \sum_{g^{c}} \phi^{k_{0}}\left(g^{c^{-1}} \cdot g\right) \cdot \delta_{k, g^{c} \cdot k_{0}}
$$

This function has the full crystal and sample symmetry according to Eq. (18b). The texture function $f(g)$ is obtained according to Eq. (6b) by:

$$
f(g)=\frac{1}{N^{c}} \sum_{g^{c}} \phi^{k_{0}}\left(g^{c} \cdot g\right)
$$

In the definition of the function $\phi^{k_{0}}$ it was assumed that the lamellae were perpendicular to the crystal direction $k_{0}$. Hence, the lamellae distribution function $L(x)$ in the sample coordinate system is the distribution of crystal directions $k_{0}$ with respect to the sample coordinate system $K_{A}$ corresponding to the crystals the orientation 
distribution of which is $\phi^{k_{0}}(g)$. This is the $k_{0}$-pole figure $P_{k_{0}}^{\prime}(x)$ of the function $\phi^{k_{0}}(g)$

$$
L(x)=\frac{1}{2 \pi} \int_{k_{0} \| x} \phi^{k_{0}}(g) d \psi=P_{k_{0}}^{\prime}(x)
$$

Since the function $\phi^{k_{0}}(g)$ does not have the full crystal symmetry the pole figures of the crystallographically equivalent directions $g^{c} \cdot k_{0}$ of this function are in general different from $P_{k_{0}}^{\prime}(x)$

$$
P_{g^{c} \cdot k_{0}}^{\prime}(x) \neq P_{k_{0}}^{\prime}(x)
$$

The $k_{0}$ pole figure of the total texture $f(g)$ defined according to Eq. (10) corresponds to the full crystal symmetry, Eq. (22)

$$
P_{\boldsymbol{g}^{c} \cdot k_{0}}(x)=P_{k_{0}}(x)
$$

Hence, in general, the lamellae distribution function $L(x)$ is not identical with the $k_{0}$-pole figure of the total texture $f(g)$

$$
P_{k_{0}}(x)=\frac{1}{N^{c}} \sum_{g^{c}} P_{g^{c} \cdot k_{0}}^{\prime}(x) \neq L^{k_{0}}(x)
$$

The function $F(g, x)$ is related to $\phi(g, k)$ according to Eq. (5b). Hence, in the specific case considered here, it is according to Eq. (32):

$$
F(g, x)=\frac{1}{N^{c}} \sum_{g^{c}} \phi^{k_{0}}\left(g^{c^{-1}} \cdot g\right) \cdot \delta_{g^{-1} \cdot x, g^{c} \cdot k_{0}}
$$

In order to obtain the general axis distribution function $D(h, y, x)$ the function $F(g, x)$ is to be integrated according to Eq. (7a) over all those orientations $g$ for which the crystal direction $h$ is parallel to the sample direction $y$. At the same time, according to Eq. (40) the function $F(g, x)$ is different from zero only if one of the crystallographically equivalent directions $g^{c} \cdot k_{0}$ is parallel to the sample direction $x$. The directions $x$ and $y$ are well defined sample directions. The angle between them be

$$
\alpha=\Varangle(x, y)
$$

Similarly the crystal directions $h$ and $g^{c} \cdot k_{0}$ are well defined directions, too, the angles between which may be

$$
\beta^{c}=\Varangle\left(h, g^{c} \cdot k_{0}\right)
$$


The integral Eq. (7a) over the function Eq. (40) is thus different from zero only if one of the angles $\beta^{c}$ is identical with the angle $\alpha$. Hence, three cases may be distinguished according to whether none of the angles $\beta^{c}$, one $\beta^{c}$, or several ones are equal to $\alpha$. In these cases the integral $D(h, y, x)$ over $F(g, x)$ takes on the forms

$$
\begin{aligned}
D(h, y, x) & =\phi\left(g_{0}\right) & & \left(\beta^{c}=\alpha\right) \\
& =0 & & \left(\beta^{c} \neq \alpha\right)
\end{aligned}
$$

where the orientation $g_{0}$ is defined by

$$
g_{0}=\left\{h\left\|y, k_{0}\right\| x\right\}
$$

and the function $\phi\left(g_{0}\right)$ has the form

$$
\phi\left(g_{0}\right)=\frac{1}{N} \sum_{l=1}^{N} \phi^{k_{0}}\left(g^{c_{i}^{-1}} \cdot g_{0}\right)
$$

The summation in Eq. (45) is to be extended over all crystal symmetry operations for which $\beta^{c}=\alpha$ is fulfilled.

\section{SERIES EXPANSIONS}

The considered functions can be developed into series of spherical harmonics (ref. Bunge, 1982):

$$
\begin{gathered}
F(g, x)=\sum_{l=0}^{\infty} \sum_{m=-l}^{+l} \sum_{n=-1}^{+l} \sum_{\lambda=0}^{\infty} \sum_{p=-\lambda}^{+\lambda} C_{l \lambda}^{m n p} T_{l}^{m n}(g) K_{\lambda}^{p}(x) \\
\phi(g, k)=\sum_{l=0}^{\infty} \sum_{m=-l}^{+l} \sum_{n=-l}^{+l} \sum_{\lambda=0}^{\infty} \sum_{p=-\lambda}^{+\lambda} Q_{l \lambda}^{m n p} T_{l}^{m n}(g) K_{\lambda}^{p}(k)
\end{gathered}
$$

whereby the symmetries have not been taken into account. With Eq. (5) and expressing $K_{\lambda}^{p}(g \cdot h)$ and $K_{\lambda}^{p}\left(g^{-1} \cdot x\right)$ by the addition theorem

$$
\begin{aligned}
K_{\lambda}^{p}(g \cdot k) & =\sum_{s=-\lambda}^{+\lambda} T_{\lambda}^{* p s}(g) \cdot K_{\lambda}^{s}(k) \\
K_{\lambda}^{p}\left(g^{-1} \cdot x\right) & =\sum_{e=-\lambda}^{+\lambda} T_{\lambda}^{s p}(g) \cdot K_{\lambda}^{s}(x)
\end{aligned}
$$


Eq. (46) can be written

$$
\begin{gathered}
F(g, g \cdot k)=\sum_{l m n \lambda p s} C_{l \lambda}^{m n p} T_{l}^{n *}(g) T_{\lambda}^{n p s}(g) K_{\lambda}^{s}(k) \\
\phi\left(g, g^{-1} \cdot x\right)=\sum_{l m n \lambda p s} Q_{l \lambda}^{m n p} T_{l}^{m n}(g) T_{\lambda}^{s p}(g) K_{\lambda}^{s}(x)
\end{gathered}
$$

If the products of the harmonics $T$ are expressed by sums and the Clebsh-Gordan coefficients these relationships take on the form

$$
F(g, g \cdot k)=\sum_{l m n \lambda p s} \sum_{l^{\prime}=|l-\lambda|}^{|l+\lambda|} C_{l \lambda}^{m n p}\left(l \lambda m p \mid l^{\prime} q\right)\left(l \lambda n s \mid l^{\prime} r\right) T_{l^{\prime}}^{q r}(g) K_{\lambda}^{s}(k)
$$

$\phi\left(g, g^{-1} \cdot x\right)=\sum_{l m n \lambda p s} \sum_{l^{\prime}=|l-\lambda|}^{|l+\lambda|} Q_{l \lambda}^{m n p}\left(l \lambda m p \mid l^{\prime} q\right)\left(l \lambda n s \mid l^{\prime} r\right) T^{q r}(g) K_{\lambda}^{s}(x)$

Comparison with Eq. (46) gives the relationships between the coefficients

$$
\begin{aligned}
& C_{l_{\lambda}^{\prime r}}^{q s}=\sum_{l m n p} Q_{l \lambda}^{m n p}\left(l \lambda m p \mid l^{\prime} q\right)\left(l \lambda n s \mid l^{\prime} r\right) \\
& Q_{l_{\lambda}^{\prime r s}}^{q r s}=\sum_{l m n p} C_{l \lambda}^{m n p}\left(l \lambda m p \mid l^{\prime} q\right)\left(l \lambda n s \mid l^{\prime} r\right)
\end{aligned}
$$

According to Eq. (6) and Eq. (46) the texture takes on the form

$$
f(g)=\sum_{l=0}^{\infty} \sum_{m=-l}^{+l} \sum_{n=-l}^{+l} C_{l}^{m n} T_{l}^{m n}(g)
$$

with the coefficients

$$
C_{l}^{m n}=\frac{1}{\sqrt{4 \pi}} C_{l 0}^{m n 0}=\frac{1}{\sqrt{4 \pi}} Q_{l 0}^{m n 0}
$$

The lamellae distribution functions can be expressed

$$
\begin{aligned}
& L(x)=\sum_{\lambda=0}^{\infty} \sum_{p=-\lambda}^{+\lambda} C_{0 \lambda}^{00 p} K_{\lambda}^{p}(x) \\
& \Lambda(k)=\sum_{\lambda=0}^{\infty} \sum_{p=-\lambda}^{+\lambda} Q_{0 \lambda}^{00 p} K_{\lambda}^{p}(k)
\end{aligned}
$$


The particular axis distribution functions are expanded in the form

$$
\begin{aligned}
& D(h, y, x)=\sum_{l m n \lambda p} \frac{4 \pi}{2 l+1} C_{l \lambda}^{m n p} K_{l}^{* m}(h) K_{l}^{n}(y) K_{\lambda}^{p}(x) \\
& \Delta(h, y, k)=\sum_{l m n \lambda p} \frac{4 \pi}{2 l+1} Q_{l \lambda}^{m n p} K_{l}^{* m}(h) K_{l}^{n}(y) K_{\lambda}^{p}(k)
\end{aligned}
$$

The total axis distribution function has the form

$$
A(h, y)=\sum_{l=0}^{\infty} \sum_{m=-l}^{+l} \sum_{n=-l}^{+l} \frac{4 \pi}{2 l+1} C_{l}^{m n} K_{l}^{* m}(h) K_{l}^{n}(y)
$$

which may be written in terms of the total pole figure

with the coefficients

$$
P_{h}(y)=\sum_{l=0}^{\infty} \sum_{n=-l}^{+l} F_{l}^{n}(h) K_{l}^{n}(y)
$$

$$
F_{l}^{n}(h)=\frac{4 \pi}{2 l+1} \sum_{m=-l}^{+l} C_{l}^{m n} K_{l}^{* m}(h)
$$

whereby the coefficients $C_{l}^{m n}$ are further expressed by Eq. (52). In the specific case of texture and lamellae distribution being independent of each other the coefficients $C$ and $Q$ take on the specific form

$$
\begin{aligned}
& C_{l \lambda}^{m n p}=C_{l 0}^{m n 0} \cdot C_{0 \lambda}^{00 p} \\
& Q_{l \lambda}^{m n p}=Q_{l 0}^{m n 0} \cdot Q_{0 \lambda}^{00 p}
\end{aligned}
$$

The specific function $\phi^{k_{0}}$, Eq. (32); may be written in the form

$$
\phi^{k_{0}}(g)=\sum_{l=0}^{\infty} \sum_{m=-l}^{+l} \sum_{n=-l}^{+l} C_{l}^{m n}\left(k_{0}\right) T_{l}^{m n}(g)
$$

with

$$
C_{l}^{m n}\left(k_{0}\right)=\sum_{\lambda=0}^{\infty} \sum_{p=-\lambda}^{+\lambda} Q_{l \lambda}^{m n p} K_{\lambda}^{p}\left(k_{0}\right)
$$

\section{THE ABSORPTION FACTOR IN A LAMELLAR SANDWICH STRUCTURE}

In order to describe X-ray diffraction in a polycrystalline, textured, lamellar eutectic, anisotropic absorption, as an essential factor, has 
to be taken into account. We consider $\mathrm{X}$-ray reflection in a two-phase material. The intensity reflected at the lattice planes perpendicular to $h$ in the phase $\alpha$ in the sample direction $y$ is given by

$$
I_{h}^{\alpha}(y)=I_{0} \cdot V^{\alpha} \cdot P_{h}^{\alpha}(y) \cdot A^{\alpha}
$$

where $I_{0}$ is the intensity of the incident beam, $V^{\alpha}$ is the volume fraction of the phase $\alpha, P_{h}^{\alpha}(y)$ is its pole figure and $A^{\alpha}$ is an absorption factor which depends on the paths of the X-rays in the two phases (Bunge, 1985)

We consider two limiting cases:

In the case of large particles, compared with the penetration depths of the X-rays, the path of rays reflected in the phase $\alpha$ is nearly exclusively in this same phase $\alpha$, Fig. 3a. This case is identical with single phase reflection. Hence, the absorption factor
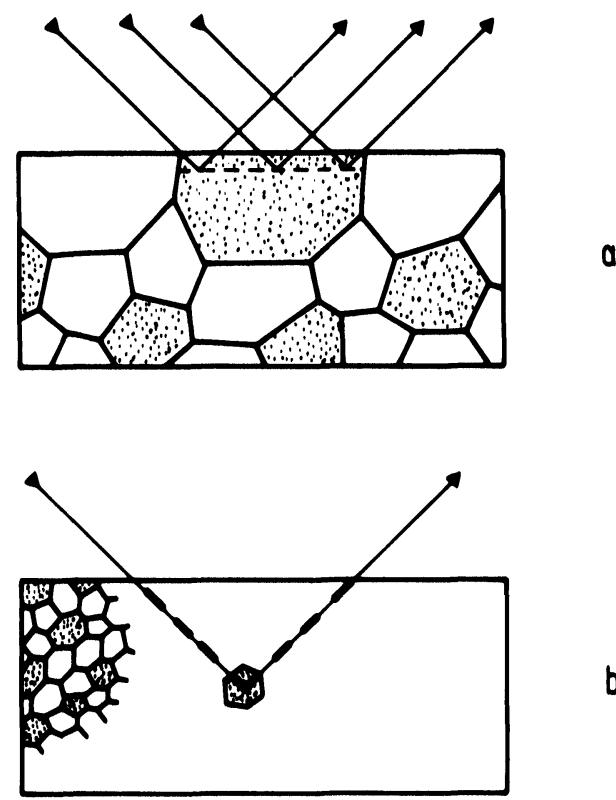

b

FIGURE 3 Reflection of X-rays in one phase of a two-phase material. (a) Large particles, (b) Small particles. 


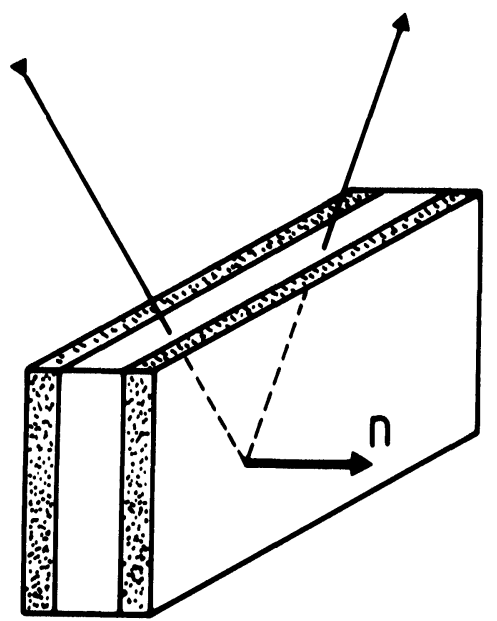

FIGURE $4 \quad \mathrm{X}$-ray diffraction with the diffraction plane parallel to the plane of the eutectic lamellae corresponds to the large particle case.

has the form

$$
A^{\alpha}=\frac{1}{\mu^{\alpha}}
$$

where $\mu^{\alpha}$ is the absorption coefficient of the phase $\alpha$.

In the case of small particles the path of rays reflected in the phase $\alpha$ is in both phases, Figure $3 \mathrm{~b}$. If the particles are small enough and do not have a preferred arrangement, we may assume in a good approximation

$$
A^{\alpha}=\frac{1}{\bar{\mu}}, \quad \bar{\mu}=V^{1} \mu^{1}+V^{2} \mu^{2}
$$

We now consider a lamellar eutectic, the lamellar diameter of which is large compared with the penetration depth, the lamellar thickness, however, is small. Then X-ray reflection corresponds to the large particle case only if the plane of the lamellae is parallel to the reflection plane, Figure 4, in all other cases it corresponds to the small particle case. 


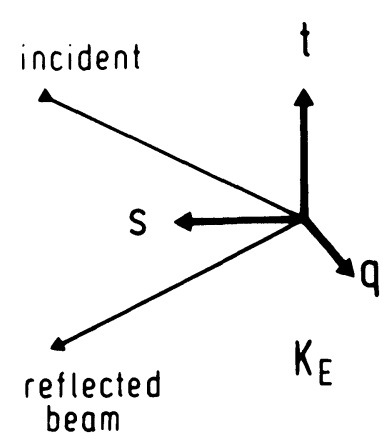

0

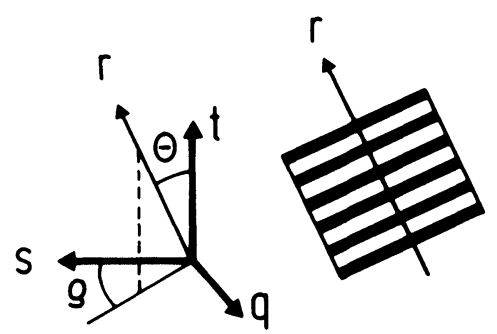

$K_{E}$

b

FIGURE 5 Definition of the experimental coordinate system $K_{E}$ (a) and an experimental direction $r$ (b) normal to the lamellae plane.

We assume an experimental coordinate system $K_{E}$, Figure 5a, made up of the diffraction direction $s$, the diffraction plane normal $t$ and a third direction $q$ perpendicular to both.

$$
K_{E}=\{s, q, t\}
$$

The normal direction $n$ to the lamellae plane may be parallel to the experimental direction $r$ expressed by the angles $\theta$ and $\rho$ in $K_{E}$, Figure $5 b$.

$$
r=\{\theta, \rho\}
$$

In the most general case the absorption factor $A^{\alpha}$ is a function of the lamellar orientation $r$ with respect to $K_{E}$. The shape of this function depends on the lamellae thicknesses $D^{\alpha}$, the respective absorption coefficients $\mu^{\alpha}$ and the Bragg angle $\vartheta$

$$
A^{\alpha}(r)=A_{D, \mu, \vartheta}^{\alpha}(\theta, \rho)
$$

A general expression for the absorption coefficient $A^{\alpha}(r)$ and numerical values will be given elsewhere.

In the specific case of large but thin lamellae and sufficiently different absorption coefficients the absorption factor can be ap- 


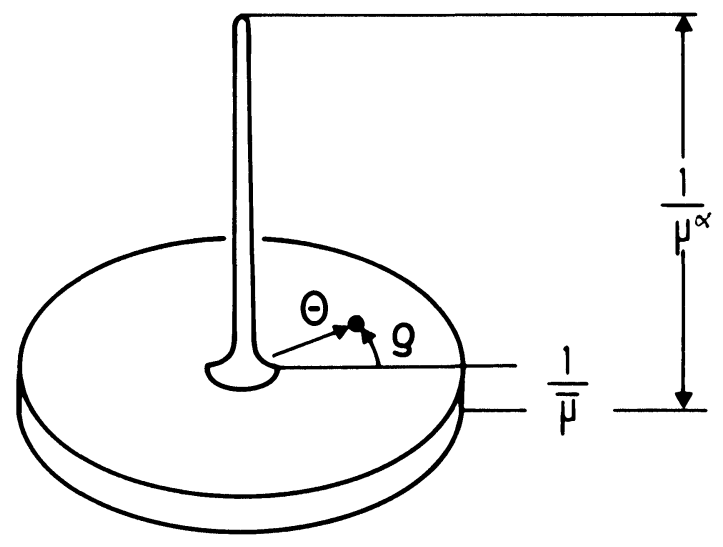

FIGURE 6 The absorption factor in the case of large but thin lamellae with sufficiently different absorption coefficients $\mu^{1}$ and $\mu^{2}$.

proximated in the form

$$
A^{\alpha}(r)=\frac{1}{\bar{\mu}}+\left(\frac{1}{\mu^{\alpha}}-\frac{1}{\bar{\mu}}\right) \delta_{r, t}
$$

which is shown schematically in Figure 6.

\section{X-RAY DIFFRACTION IN LAMELLAR EUTECTICS}

In order to describe $\mathrm{X}$-ray diffraction in polycrystalline, textured lamellar eutectics the sample orientation $\Gamma$ with respect to the experimental coordinate system $K_{E}$ has to be specified as is shown in Figure $7 \mathrm{a}$

$$
K_{A}=\Gamma \cdot K_{E}
$$

It is the rotation $\Gamma$ which transforms the experimental coordinate system $K_{E}$ into the sample coordinate system $K_{A}$. An experimental direction $r$ is then parallel to a sample direction $x$ such that

$$
r=\Gamma \cdot x, \quad r_{i}=\Gamma_{i j} \cdot x_{j}
$$

The sample orientation $\Gamma$ may be expressed by three Euler angles $\omega \chi \phi$ which are the rotation angles of the Eulerian cradle of a 

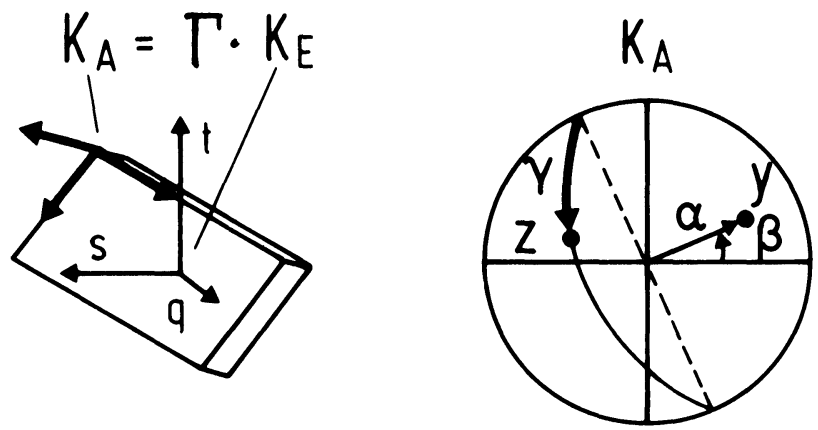

a

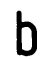

FIGURE 7 The sample orientation $\Gamma$ relative to the experimental coordinate system $K_{E}$. (a) The sample in the system $K_{E}$, (b) The directions $y$ (diffraction directions $s$ ) and $z$ (diffraction plane normal $t$ ) in the sample coordinate system, expressed by the three angles $\alpha \beta \gamma$.

texture goniometer (ref. Bunge and Puch, 1984)

$$
\Gamma=\{\omega, \chi, \phi\}
$$

Another description of the sample orientation $\Gamma$ is obtained by specifying the sample directions $y$ and $z$ parallel to the experimental directions $s$ and $t$ respectively, i.e. the diffraction direction and the diffraction plane normal, Figure $7 \mathrm{~b}$, by

$$
\begin{aligned}
& s=\Gamma \cdot y \\
& t=\Gamma \cdot z
\end{aligned}
$$

In normal pole figure measurements only the sample direction $y$ is relevant. It is expressed by the angles $\alpha, \beta$ in the sample coordinate system $K_{A}$. The direction $z$ is perpendicular to $y$. Hence, it can be fixed by a third angle $\gamma$, i.e. a rotation about the diffraction direction $y \| s$ which is irrelevant in usual pole figure measurements. Hence, the sample orientation can also be expressed by the three angles $\alpha \beta \gamma$ or the two directions $y, z$.

$$
\Gamma=\{y, z\}=\{\alpha, \beta, \gamma\}
$$

as is shown in Figure $7 b$. 
With an absorption factor according to Eq. (66) the reflected X-ray intensity, Eq. (61), can be written more precisely using the particular pole figure instead of the total one

$$
I_{h}^{\alpha}(\Gamma)=I_{0} \cdot V^{\alpha} \cdot \int D^{\alpha}(h, y, x) \cdot A^{\alpha}(\Gamma \cdot x) d x
$$

With the specific form of the absorption factor according to Eq. (67) this expression takes on the form

$$
I_{h}^{\alpha}(\Gamma)=I_{0} \cdot V^{\alpha}\left[\frac{1}{\bar{\mu}} P_{h}^{\alpha}(y)+\left(\frac{1}{\mu^{\alpha}}-\frac{1}{\bar{\mu}}\right) D^{\alpha}(h, y, z)\right]
$$

The reflected intensity depends on the full sample orientation $\Gamma$ and not only on two orientation angles $\alpha, \beta$ as in the case of the usual pole figures. Hence, in the case of $\mathrm{X}$-ray diffraction in polycrystalline, textured lamellar eutectics the usual two-dimensional pole figures are to be replaced by generalized three-dimensional pole figures such that

$$
I_{h}^{\alpha}(\Gamma)=I_{0} \cdot V^{\alpha} \cdot Q_{h}^{a}(\Gamma)
$$

where the sample orientation $\Gamma$ may be expressed according to Eq. (70) or to Eq. (72). This latter form shows that the threedimensional pole figures depend on a rotation of the sample about the diffraction direction $s$ which is not the case in the usual pole figures, especially those of single phase materials.

The experimental arrangement. (Figure 5) exhibits a certain symmetry $G^{e}$ with the operations $g^{e}$ and $u^{e}$ symbolized by three dots

$$
G^{e}=\left\{g^{e}, u^{c}\right\},
$$

This symmetry depends on the experimental conditions i.e. whether $\mathrm{X}$-ray diffraction is based on normal or anomalous scattering. In these cases it is

$$
\begin{aligned}
G^{e} & =D_{2 h}=m m m & & \text { normal scattering } \\
& =C_{2 v}=2 m m & & \text { anomalous scattering }
\end{aligned}
$$

In the case of anomalous scattering the two-fold axis is parallel to the diffraction vector $s$ and in the case of normal scattering an inversion centre is added, Figure 8.

Since the sample also has a symmetry described in Eq. (15), the 

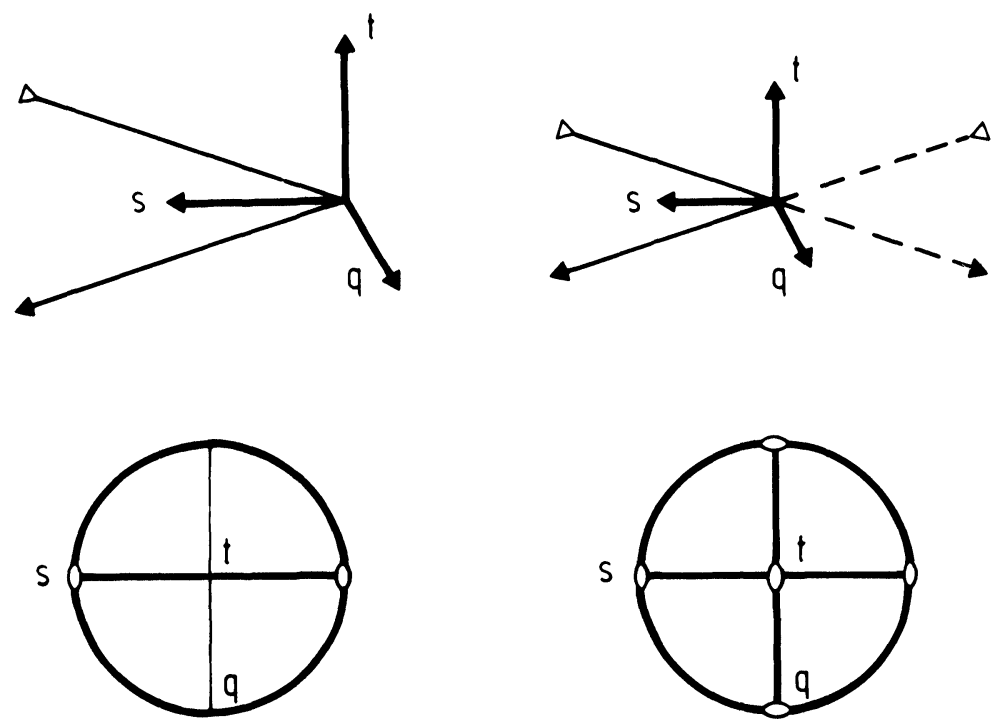

$C_{2 V}=2 \mathrm{~mm}$

$D_{2 h}=m m m$

FIGURE 8 Experimental symmetry in the case of normal diffraction, $\mathrm{mmm}$ and anomalous diffraction, $2 \mathrm{~mm}$.

three-dimensional pole figure $Q_{h}(\Gamma)$ Eq. (75) has the symmetries

$$
\dot{\bar{Q}}_{h}\left(g^{s} \cdot \Gamma \cdot g^{e}\right)=\dot{\hat{Q}}_{h}(\Gamma)
$$

The three-dimensional pole figure as a function of the sample orientation $\Gamma$ with respect to the experimental coordinate system $K_{E}$ must not be mixed up with the three-dimensional orientation distribution function (ODF) which is a function of the crystal orientation $g$ with respect to the sample coordinate system $K_{A}$.

In the specific case of lamellae orientation independent of the texture, Eq. (73) is transformed by Eq. (27a) into

$$
I_{h}^{\alpha}(\Gamma)=I_{0} \cdot V^{\alpha} \cdot P_{h}^{\alpha}(y) \cdot \int L(x) \cdot A^{\alpha}(\Gamma \cdot x) d x
$$

With Eq. (67) this takes on the form

$$
I_{h}^{\alpha}(\Gamma)=I_{0} \cdot V^{\alpha} \cdot P_{h}^{\alpha}(y) \cdot\left[\frac{1}{\bar{\mu}}+\left(\frac{1}{\mu^{\alpha}}-\frac{1}{\bar{\mu}}\right) \cdot L(z)\right]
$$


This means, each pole figure is multiplied by the same lamellae distribution factor.

An even more specific case is obtained if the lamellae are perpendicular to one specific sample direction $x_{0}$ which may then be taken as the $x_{3}$ direction. This case is obtained, for instance, if textured foils of two different materials are stacked upon each other such as to form an "artificial eutectic". In this case Eq. (79) takes on the form

$$
I_{h}^{\alpha}(\Gamma)=I_{0} \cdot V^{\alpha} \cdot P_{h}^{\alpha}(y) \cdot A^{\alpha}(r)
$$

where $r=\{\theta, \rho\}$ is the normal direction $x_{3}$ of the sample expressed in the experimental coordinate system $K_{E}$.

Equation (81) can be used to determine the absorption factor for lamellar structures experimentally. With the absorption factor Eq. (67) finally this takes on the form

$$
I_{h}^{\alpha}(\Gamma)=I_{0} \cdot V^{\alpha} \cdot P_{h}^{\alpha}(y) \cdot\left[\frac{1}{\bar{\mu}}+\left(\frac{1}{\mu^{\alpha}}-\frac{1}{\bar{\mu}}\right) \delta_{x_{3}, t}\right]
$$

The absorption factor was measured experimentally in a "synthetic eutectic" prepared by stacking aluminium and copper foils upon each other. This fulfills the two conditions mentioned above that texture and lamellae orientation are independent of each other and that the lamellae are perpendicular to a specific sample direction. A cross section through the stack is shown in Figure 9. The lamellae

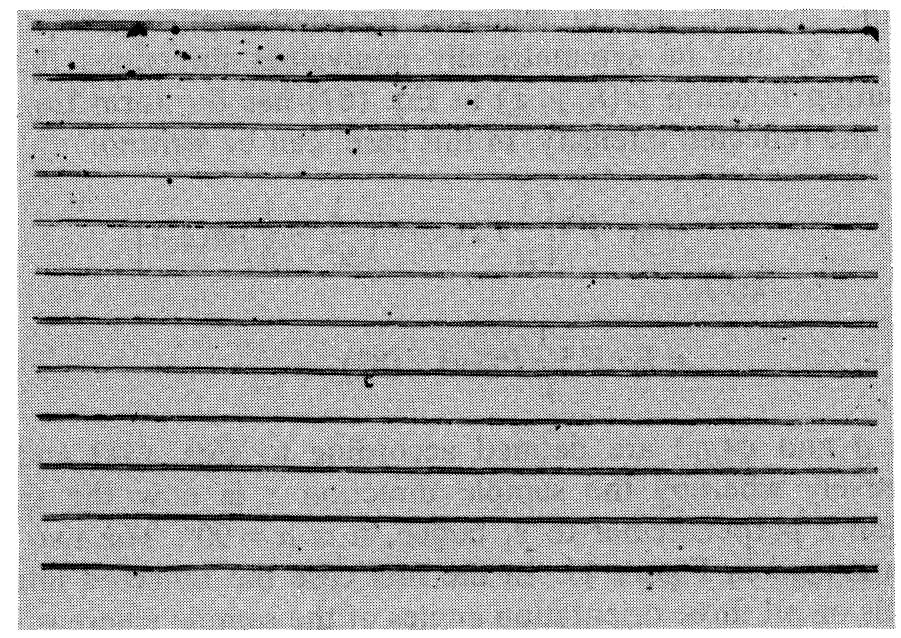

FIGURE 9 Cross-section through a "synthetic eutectic", i.e. a stack of aluminium and copper foils. 
TABLE I

The lamellae thicknesses, linear absorption coefficients and penetration depths for $\mathrm{MoK}_{\alpha^{-}}$ radiation for the sandwich sample Figure 9

\begin{tabular}{cccc}
\hline & $D^{\alpha}[\mu \mathrm{m}]$ & $\mu^{\alpha}\left[\mathrm{cm}^{-1}\right]$ & $\begin{array}{c}\text { Penetration depth } \\
1 / \mu^{\alpha}[\mu \mathrm{m}]\end{array}$ \\
\hline Al & 31 & 14.3 & 699 \\
$\mathrm{Cu}$ & 3.4 & 444 & 22.5 \\
\hline
\end{tabular}

thicknesses, absorption coefficients and penetration depths are shown in Table I. X-ray diffraction measurements were carried out at the five-circle diffractometer in the HASYLAB at DESY in Hamburg because of the small angular divergence of the X-ray beam available with this equipment (Kupcik et al. 1983). The absorption coefficients correspond to $\mathrm{MoK}_{\alpha}$-radiation. Measurements were carried out with the sample normal direction (which is perpendicular to the foil normal direction) parallel to the diffraction direction $s$. The angle $\gamma$ is a rotation of the sample about this direction whereby in the orientation $\gamma=0$ the direction $q$ is parallel to the lamellae plane. The measured intensity of the $\mathrm{Al}(200)$ reflection is shown in Figure 10. Hence, the form of the absorption factor is, in this case, very near to that assumed in Eq. (67) and Figure 6.

In the specific case of lamellae perpendicular to one crystal direction $k_{0}$ and its symmetrically equivalent directions the axis distribution function $D(h, y, z)$ in Eq. (67) has the form Eq. (43). Hence the reflected intensity, in this case, can be written:

$$
\begin{aligned}
& =I_{0} \cdot V^{\alpha}\left[\frac{1}{\bar{\mu}} P_{h}^{\alpha}(y)+\left(\frac{1}{\mu^{\alpha}}-\frac{1}{\bar{\mu}}\right) \phi\left(g_{0}\right)\right] \\
& =I_{0} \cdot V^{\alpha} \frac{1}{\bar{\mu}} P_{h}^{\alpha}(y)
\end{aligned}
$$

where $q$ and $\phi\left(g_{0}\right)$ are defined according to Eqs. (44) and (45) respectively whereby the sample direction $x$ is now the specific direction $z$ perpendicular to $y$, hence, it is $\alpha=90^{\circ}$. The two cases are illustrated in Figure 11. In the first case the reflected intensity depends on all three orientation angles of the sample whereas in the second case it depends only on the usual pole figure angles $\alpha, \beta$ and 


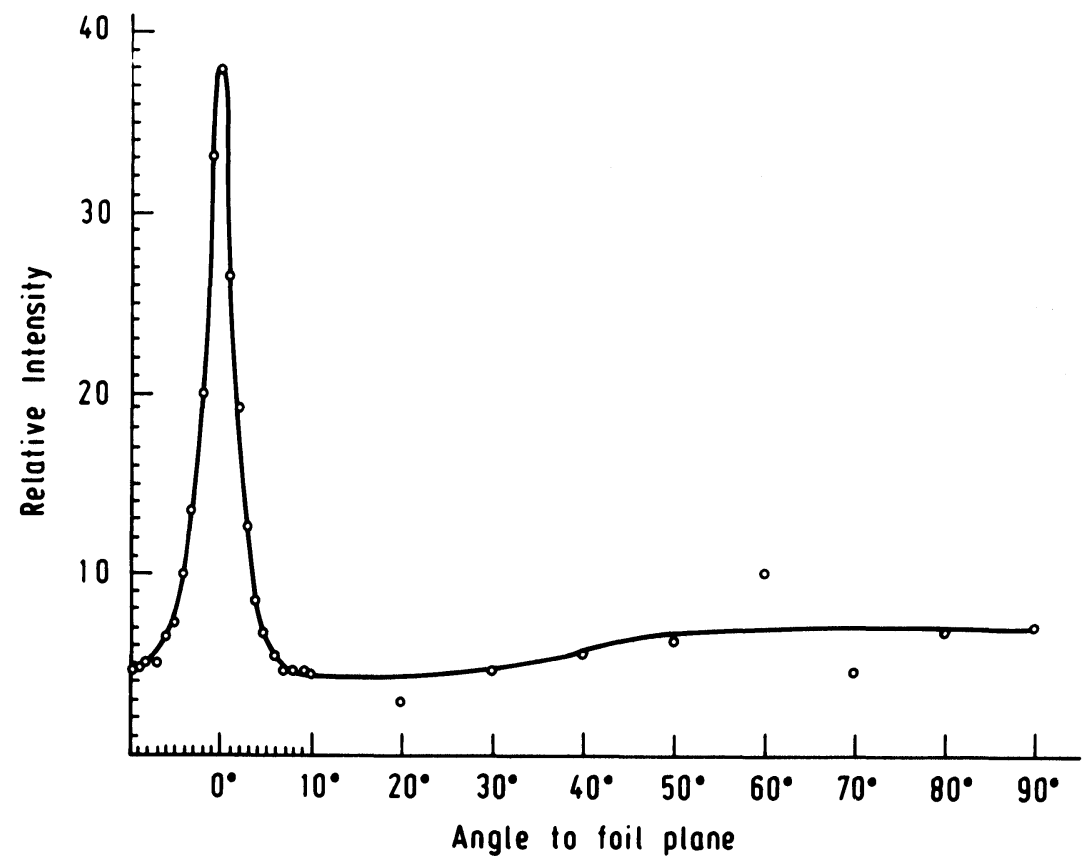

FIGURE 10 The absorption factor for $\mathrm{MoK}_{\alpha}$-radiation measured in the "synthetic eutectic", Figure 9, as a function of the rotation angle $\gamma$ about the sample normal direction.
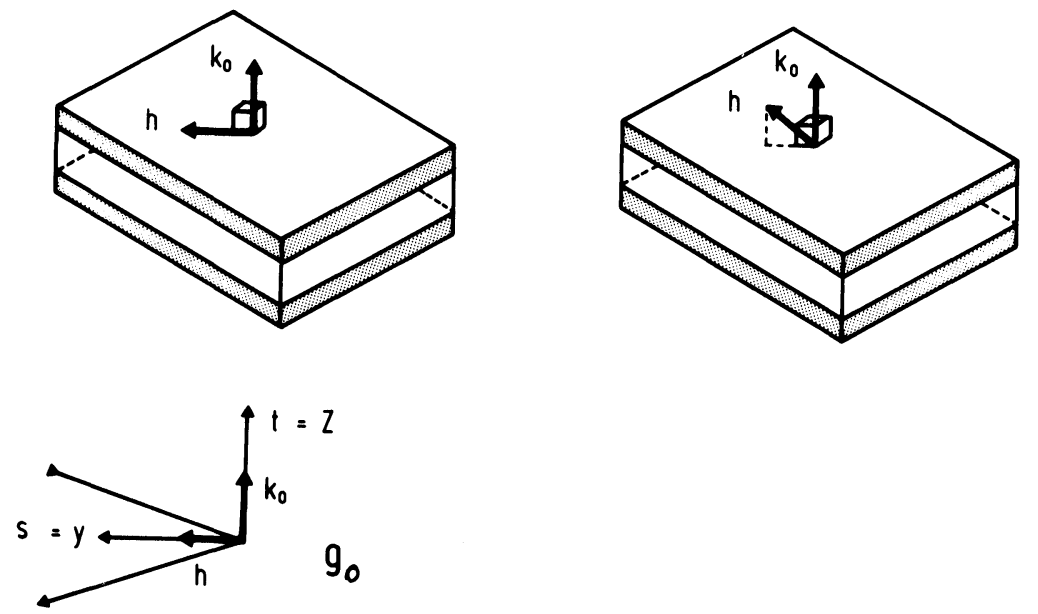

FIGURE 11 The lamellae normal direction is parallel to the crystal direction $k_{0}$. The normal direction $h$ to the reflecting lattice, plane is: (a) perpendicular to $k_{0}$. Definition of the orientation $g_{0}$, (b) not perpendicular to $k_{0}$. 
not on the rotation $\gamma$ about the diffraction vector $s$. If a threedimensional dependence of the intensity according to Eq. (83a) is found for several pole figures $h_{i}$ then the crystallographic orientation $k_{0}$ of the lamellae normal direction can easily be determined by the condition:

$$
k_{0} \perp h_{i} \quad(\text { all } i)
$$

\section{CONCLUSIONS}

In order to describe the orientation distribution in lamellar eutectics or eutectoids it is necessary to consider the crystallographic orientation of the crystallites and the geometrical orientation of the lamellae in both phases. In the most general case, the distributions of these two orientations may be interdependent. It is then necessary to define two combined orientation-shape distribution functions which depend on five orientation angles. The fivedimensional orientation distribution functions may be written in two forms, with the lamellae orientation referred either to the crystal coordinate system $K_{B}$ or to the sample coordinate system $K_{A}$. The textures, in the conventional definition, and the lamellae distribution functions are only three-dimensional or two-dimensional projections, respectively, of these five-dimensional distribution functions. Textures and lamellae distribution functions together are thus, in general, nott sufficient to describe the orientation relations in lamellar eutectics completely.

The reflection of X-rays in eutectic alloys may be influenced by the crystallographic texture as well as by the lamellae orientation if the penetration depth of the X-rays is large compared with lamellae thickness and small compared with their planar dimensions. In this case the different absorption of the X-rays in the two phases may give rise to a macroscopically anisotropic absorption factor. The reflected X-ray intensity then depends on the lamellae distribution additionally to the usual dependence on the crystallographic orientation distribution, i.e. the texture. As a consequence of this anisotropic absorption the reflected intensity may depend on a rotation of the sample about the direction of the diffraction vector $s$. This is quite different from usual pole figure measurements where 
the reflected intensity depends only on the orientation of the diffraction vector $s$ (the bisectrix between incident and reflected beam) relative to the sample coordinate system which is usually described by the two pole figure angles $\alpha, \beta$. In the case of eutectic alloys it is thus necessary to measure three-dimensional generalizations of the usual two-dimensional pole figures which depend also on a third angle $\gamma$ i.e. a rotation about the diffraction vector $s$. These three-dimensional pole figures depend on a complete fivedimensional orientation-lamellae-distribution. This provides the possibility to obtain information about the lamellae orientation distribution function of which, by optical methods, only a planar section function can be obtained. It also provides information about the crystallographic orientations of lamellae planes and their respective distribution which is otherwise difficult to obtain.

\section{References}

Bunge, H. J. (1982) Texture Analysis in Materials Science. Butterworths, London. Bunge, H. J. (1985) Z. Metallkde. 76, 92-101.

Bunge, H. J. and Puch, K. H. (1984) Z. Metallkde. 75, 124-132.

Kurz, W. and Sahm, P. R. (1975) Gerichtet erstarrte eutektische Werkstoffe. Springer, Berlin.

Kupčik, V., Wulf, R., Wendschuh, M., Wolf, A. and Pähler, A. (1983) Nuclear Instr. and Meth. 208, 519-522. 